\title{
Circadian Rhythm in Membrane Conductance Expressed in Isolated Neurons
}

\author{
Stephan Michel, Michael E. Geusz, Joshua J. Zaritsky, \\ Gene D. Block*
}

Although isolated neurons can generate rhythmic activity, they have not yet been shown to generate rhythms with a period in the circadian range (near 24 hours). The eye of the mollusk Bulla gouldiana expresses a circadian rhythm in optic nerve impulses that is generated by electrically coupled cells known as basal retinal neurons (BRNs). Daily fluctuations in the membrane potential of the BRNs appear to be driven by a rhythm in membrane conductance. Isolated BRNs exhibited spontaneous conductance changes similar to those observed in the intact retina. Membrane conductance was high in the late subjective night and decreased approximately twofold near projected dawn during at least two circadian cycles in culture. The persistence of daily conductance changes in isolated BRNs indicates that individual neurons can function as circadian pacemakers.

Circadian pacemakers have been localized within the nervous system of a number of multicellular organisms (1), yet it is still not certain whether individual neurons have the capacity to generate circadian rhythms or whether the rhythmicity requires the interaction of cells within tissues. Perhaps the strongest evidence to date for cellular autonomy of circadian rhythmicity among the Metazoa comes from dispersed avian pinealocytes, which express a rhythm in melatonin release for several cycles in culture (2). We now report that isolated cultured neurons from the molluskan retina exhibit circadian rhythms.

The eyes of several mollusks contain circadian pacemakers that generate rhythms in the frequency of compound action potentials (CAPs) recorded in vitro from the optic nerve (3). In the eye of Bulla gouldiana, CAPs are generated by the synchronous firing of about 100 electrically coupled BRNs. Earlier workers localized the circadian pacemaker to the base of the retina and predicted that each $B R N$ should function as an individual circadian pacemaker if it is physically isolated from the remaining cells $(4,5)$. The present study tests this hypothesis by monitoring membrane conductance at several phases of the circadian cycle.

Changes in BRN membrane conductance are most pronounced at projected dawn and dusk. The membrane conductance of the BRNs decreases by about half (6) as the CAP frequency increases at projected dawn from a hyperpolarized, quiescent state (5). A corresponding return to a higher conductance state occurs near projected dusk.

To compare dissociated cells with BRNs

National Science Foundation Center for Biological Timing, Department of Biology, University of Virginia, Charlottesville, VA 22901.

*To whom correspondence should be addressed.

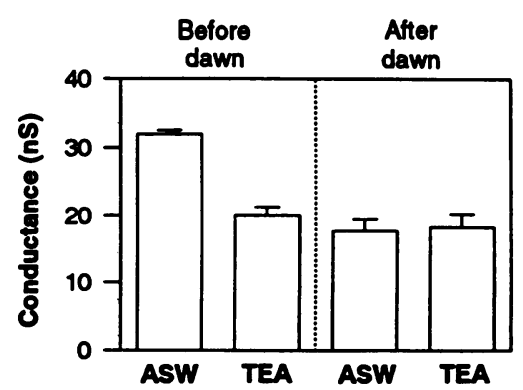

Fig. 2. Effect of the $\mathrm{K}^{+}$channel blocker TEA on the membrane conductance of BRNs before ( $n$ $=6)$ and after $(n=5)$ projected dawn measured in the semi-intact retina. Shown are means of the membrane conductance for BRNs before and after superfusion with $100 \mathrm{mM}$ TEA substituted for $\mathrm{NaCl}$ in ASW. Application of TEA caused a significant decrease in membrane conductance before projected dawn (ZT 19): ASW, $31.9 \pm 0.60 \mathrm{nS}$ (SEM); TEA, $20.0 \pm 1.25$ $\mathrm{nS}(P<0.001$, paired $t$ test). After projected dawn (ZT 2) TEA did not cause a significant decrease in conductance: ASW, $17.7 \pm 1.74$ $\mathrm{nS}$; TEA, $18.3 \pm 1.92 \mathrm{nS}(P>0.5)$.

TEA-sensitive channels were now largely closed.

To determine whether dispersed BRNs continue to exhibit circadian changes in membrane conductance, we measured conductance during the first two circadian cycles after cell dispersal (Fig. 3, A through D). Because long-term recordings from BRNs in cell culture are not yet possible, we sampled the membrane conductance of BRNs throughout the circadian cycle at the phases indicated in Fig. 3E. tance (Fig. 2) but had no effect when applied after dawn, suggesting that the

The circadian conductance changes pear to be due primarily to a $\mathrm{K}^{+}$curren sensitive to the $\mathrm{K}^{+}$channel blocker tetraethylammonium chloride (TEA). Application of TEA before projected dawn led to a tion of TEA before projected dawn led to a
Fig. 1. Change in membrane conductance of two BRNs of semi-intact eye preparations (7) during projected dawn and dusk. Current pulses of 6-s duration were given from -1.2 to $0.6 \mathrm{nA}$ while in the discontinuous current clamp mode with the membrane potential brought to -65 $\mathrm{mV}$. The data for positive current pulses were excluded from the analysis because action potentials hampered measurements of membrane potential change. (A) Change in membrane potential of one BRN in response to current pulses administered at different Zeitgeber times (ZT) through projected dawn. (B) Membrane potential re-

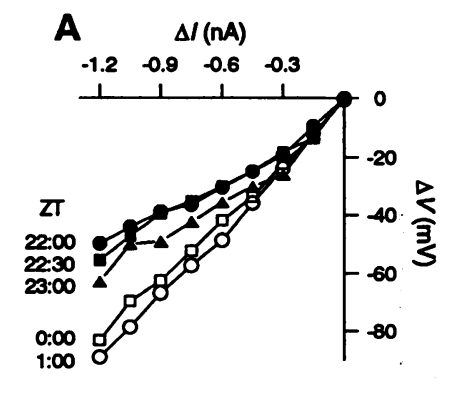

B
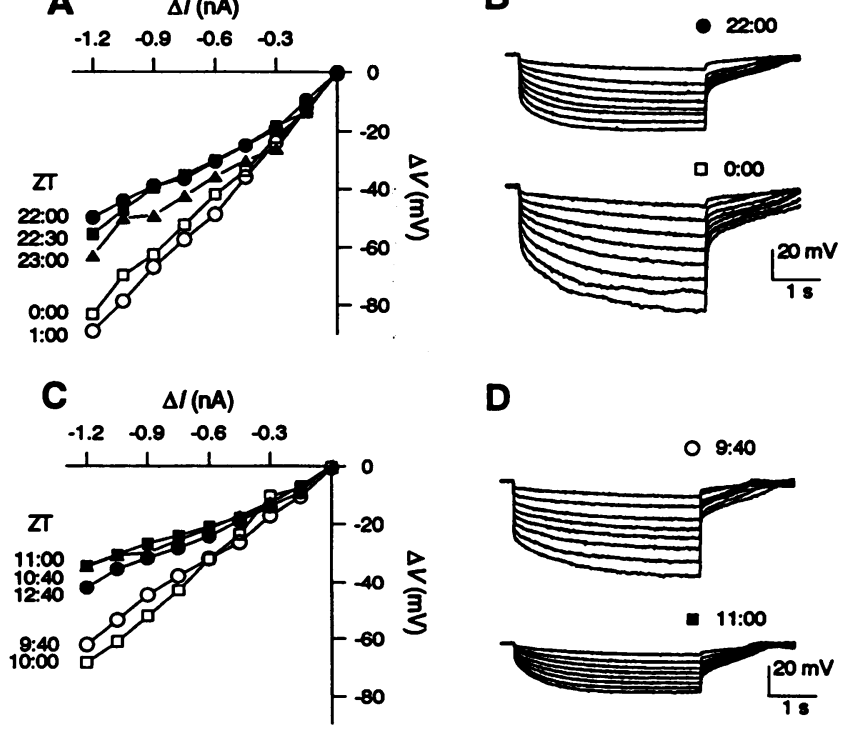

D

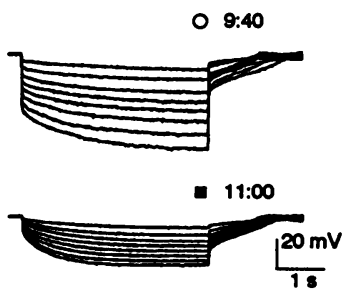

cordings during eight current pulses given before (above) and after (below) the decrease in conductance at projected dawn. Symbols indicate the points during the record used for constructing the current-voltage (I-V) curves in (A). (C) Membrane conductance measurements of one BRN made through projected dusk. Conductance increases about 1 hour before projected dusk (between ZT 10:00 and 10:40). (D) Change in membrane potential due to current pulses given before (above) and after (below) projected dusk, corresponding to the I-V curves shown in (C). 
Fig. 3. Membrane conductance of dispersed BRNs during the first two circadian cycles in culture. Two basal retinae were treated with $0.01 \%$ protease (Sigma) in ASW for 1 hour at $22^{\circ} \mathrm{C}$, rinsed in medium (11), and then triturated with fire-polished pipettes onto a glass cover slip coated with poly-o-lysine (Sigma) placed in a culture dish. Cells were maintained in dim fluorescent room light $(5 \mu \mathrm{W} /$ $\mathrm{cm}^{2}$ ) at $22^{\circ} \pm 1^{\circ} \mathrm{C}$. We removed eyes from animals at ZT 12 (group 1) or ZT 18 (all other groups) to control for any effects of duration in culture or time of dissection. BRNs were identified by morphological criteria (12). Discontinuous current clamp was used as in Fig. 1, but with the cells mounted on an inverted microscope and per-

fused with ASW. Current pulses from -0.8 to $0.4 \mathrm{nA}$ of 0.2 -s duration were given after the membrane potential reached a stable value (13). (A) Change in membrane potential in response to current pulses before (group 1, ZT 19 to 22; $n=4$ ) and after (group 2, ZT 2 to $5 ; n=7$ ) first projected dawn in culture. Error bars are SEM. The change in slope of the curves represents a significant change in conductance from group 1, $21.3 \pm 4.97 \mathrm{nS}$, to group 2, $10.7 \pm 0.98 \mathrm{nS}(P<0.005)(14)$. (B) Representative membrane potential responses to current pulses before (above) and after (below) first projected dawn. (C) Membrane conductance of BRNs increased before projected dusk from group 3, $11.5 \pm 1.07 \mathrm{nS}$ (ZT 10 to 12; $n=5)$, to group 4, $22.0 \pm 1.65 \mathrm{nS}(Z \mathrm{~T} 14$ to $16 ; n=4)(P<$ 0.005). (D) The membrane conductance decreased at the second projected dawn from group $5,11.9$ $\pm 1.24 \mathrm{nS}$ (ZT 19 to $22 ; n=6)$, to group $6,8.24 \pm 0.89 \mathrm{nS}(Z T 2$ to $4 ; n=6)(P<0.05)$. (E) Rhythm in CAP frequency in an intact Bulla eye recorded in medium (11) under constant light at room temperature. Numbers indicate the recording times for the dispersed cells and correspond to the curves in (A) through (D). The vertical dotted lines mark projected dawn (ZT 0).
As with BRNs in the retina, dispersed BRNs showed a statistically significant decrease in membrane conductance at projected dawn (Fig. 3, A and B) and a significant conductance increase at projected dusk (Fig. 3C). A conductance decrease was also observed at projected dawn on the second circadian cycle (Fig. 3D), demonstrating that the circadian rhythm in dispersed cells persists under constant conditions in cell culture.

Although dispersed BRNs were not in contact with one another and were plated at very low density (about 10 to 20 cells per dish), one could not entirely exclude the possibility of secretory interaction among the neurons. It is unlikely that secretion is required for rhythm generation in the intact retina, as the CAP rhythm persists at low $\left(10^{-7} \mathrm{M}\right)$ extracellular $\mathrm{Ca}^{2+}$ concentration (8). To confirm that the conductance changes we observed in dispersed cells were due only to their intrinsic rhythmicity, we measured the membrane conductance of BRNs cultured in complete physical isolation. The cells were cultured in microwell dishes containing one cell per well (Fig. 4). Our recordings from completely isolated neurons confirm the results obtained in low-density dispersed cell culture. Completely isolated neurons continue to exhibit significant decreases in conductance near projected dawn during two cycles of isolation (Fig. 4, A and B). Like the dispersed cells, isolated cells showed a trend toward increased conductance over time in culture. However, membrane conductance of BRNs maintained in culture for the same length of time still differed significantly before and after the second projected dawn (see curve $4^{\prime}$ of Fig. 4B).

Our results provide the strongest evi-
Fig. 4. Membrane conductance of completely isolated BRNs during two circadian cycles in culture. All culture procedures on isolated cells were performed during the subjective day. At ZT 1, the BRNs were dispersed into microwells (Terasaki dish, Nunc). After 1 hour, the dish was rinsed with medium to remove unattached cells. After 6 to 8 hours, cells were removed with a glass suction pipette and a microminipulator, leaving only one BRN per well. Constant culture conditions and conductance measurements were as in Fig. 3, except that only pulses from -0.3 to $0.1 \mathrm{nA}$ were given. (A) Change in membrane potential in response to current pulses before (group $1, Z T 19$ to $21 ; n=11$ ) and after (group 2, ZT 3 to $5 ; n=15$ ) the first projected dawn in culture (15). Error bars are SEM. The decrease in membrane conductance from $6.76 \pm 1.00 \mathrm{nS}$ to $4.30 \pm 0.30 \mathrm{nS}$ was significant $(P<0.005)(14)$. (B) Current-voltage curves of isolated BRNs after 2 days in culture measured at the same Zeitgeber times as in (A). There was a significant decrease in membrane conductance at the second projected dawn $(P<0.005)$ from $4.39 \pm 0.58 \mathrm{nS}$ (group $3, n=12)$ to 2.84 $\pm 0.16 \mathrm{nS}$ (group $4, n=7$ ). Additional BRNs were dispersed 6 hours later (ZT 7) and conductance measurements were made at the usual time after the second projected dawn (group $4^{\prime}, Z T 3$ to $5 ; n=10$ ). The membrane conductance for group $4^{\prime}$ is significantly lower $(P<0.05$; mean, $3.24 \pm 0.25)$ than for group 3 , the before-dawn group, but not different from that for group 4 , the after-dawn group that was in culture for an additional 6 hours $(P>$ 0.15). (C) Circadian rhythm in CAP frequency of an intact Bulla eye as described in Fig. 3E. The numbers indicate the recording times for the isolated cells.
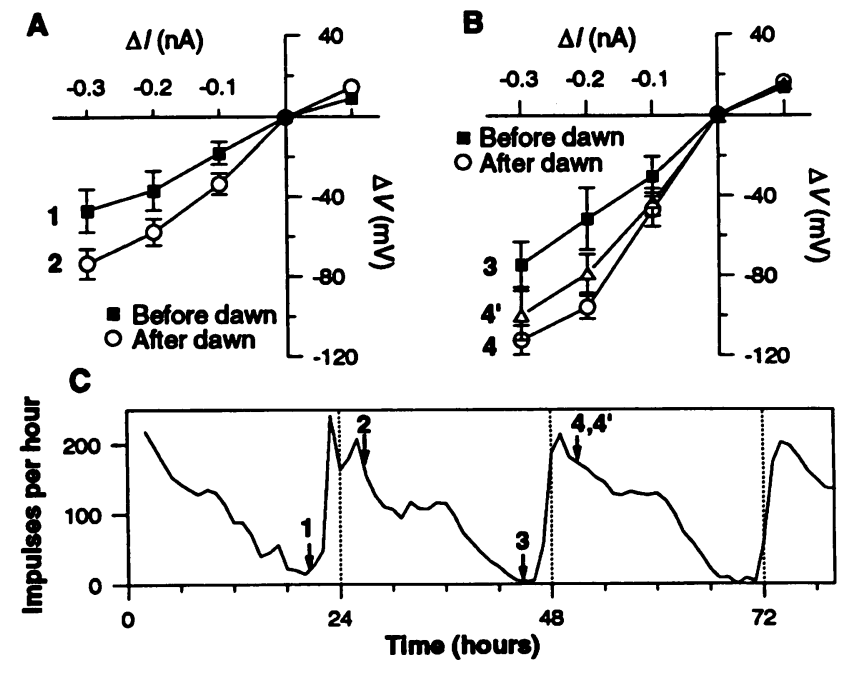
dence to date that individual neurons have the capacity to generate circadian rhythms. Cultured hypothalamic neurons of mammals show a circadian rhythm in the release of vasopressin (9), but the rhythm was measured after sufficient time for functional connections to be restored. Thus it is not yet certain whether individual hypothalamic neurons have the capacity to generate circadian rhythms.

Although we have demonstrated that individual neurons can generate a circadian rhythm, it is not clear whether isolated neurons have all of the characteristics associated with the "biological clock" in the intact retina (for example, entrainment by Zeitgebers, temperature compensation). Indeed, we failed to observe light responses from BRNs in culture, even though BRNs in the intact retina depolarize upon illumination and can be phase-shifted (10). In addition, we only rarely observed impulse production in dispersed cells. Regardless of whether some properties of circadian systems require organized tissue, our experiments confirm that the mechanisms for generation and expression of circadian rhythmicity can be present within individual neurons. This demonstration should greatly aid experimental efforts to understand the cellular basis of circadian rhythm generation by facilitating the use of biophysical and biochemical techniques with identified circadian pacemaker cells.

\section{REFERENCES AND NOTES}

1. F. Turek, Annu. Rev. Physiol. 47, 49 (1985); J. Takahashi, N. Murakami, S. S. Nikaido, B. L. Pratt, L. M. Robertson, Recent Progr. Horm. Res. 45, 279 (1989); J. W. Jacklet, in Neuronal and Cellular Oscillators, J. W. Jacklet, Ed. (Dekker, New York, 1989), pp. 483-527.

2. T. Deguchi, Nature 282, 94 (1979); L. M. Robertson and J. S. Takahashi, J. Neurosci. 8, 12 (1988); M. Zatz, D. A. Mullen, J. R. Moskal, Brain Res. 438, 199 (1988)

3. J. W. Jacklet, Science 164, 562 (1969); A. Eskin, Fed. Proc. Fed. Am. Soc. Exp. Biol. 38, 2573 (1979); and E. Harcombe, Comp. Biochem. Physiol. A 57, 443 (1977); G. D. Block and M. H. Roberts, J. Comp. Physiol. A 142, 403 (1981).

4. G. D. Block and S. F. Wallace, Science 217, 155 (1982); G. D. Block and D. G. McMahon, J. Comp. Physiol. A 155, 387 (1984).

5. D. G. McMahon, S. F. Wallace, G. D. Block J. Comp. Physiol. A 155, 379 (1984).

6. D. G. McMahon, thesis, University of Virginia (1986); M. R. Ralph and G. D. Block, J. Comp. Physiol. A 166, 589 (1990). Previous conductance measurements were made between Zeitgeber time (ZT) 19 and 6 or between 10 and 17 . The only observed transitions were at projected dawn or dusk. Zeitgeber time refers to the time during a 24-hour cycle in which ZT 0 equals projected dawn, the hour at which the light onset would have occurred if the animals had remained on the light-dark cycle.

7. D. G. McMahon and G. D. Block, J. Comp. Physiol. A 161, 335 (1987). The recording dish was continuously perfused with artificial seawate (ASW). Thin-wall glass electrodes (20 to 40 megohms) were used

8. S. B. Khalsa, M. R. Ralph, G. D. Block, Soc.
Neurosci. Abstr. 17, 1240 (1991).

9. N. Murakami et al., Brain Res. 545, 347 (1991).

10. G. D. Block and D. G. McMahon, ibid. 265, 134 (1983); M. E. Geusz and T. L. Page, J. Comp. Physiol. A 168, 565 (1991).

11. Leibovitz L-15 medium (Sigma, ICN) was modified according to the procedure of $S$. Schacher and E. Proshansky [J. Neurosci. 3, 2403 (1983)] with hemolymph omitted and gentamicin added $(0.1 \mathrm{mg} / \mathrm{ml}$, Sigma). Osmolarity was corrected to that of ASW, and pH was 7.4 to 7.8. Intact eyes maintained in the medium showed circadian rhythms with normal period and phase.

12. The criteria consisted of a cell diameter less than $50 \mu \mathrm{m}$, the presence of large organelle-like structures not found in the photoreceptors, and the lack of pigment granules associated with cells of the distal retina [J. W. Jacklet and W. Colquhoun, J. Neurocytol. 12, 673 (1983)]. The large inclusions characteristic of BRNs are evident after dye-fills of cells identified positively as BRNs in eye preparations [M. E. Geusz and G. D. Block, J. Biol. Rhythms 7, 255 (1992)]

13. Criteria for stability consisted of a resting potential between -25 and $-65 \mathrm{mV}$, no more than 5-mV drift between first and last current pulses. and no regions of negative slope of the currentvoltage curves. Cells that did not show at least a 5-mV change in membrane potential due to an injected current of $-0.2 \mathrm{nA}$ were rejected as having poor membrane sealing. We attempted recordings from 290 cells from 114 retinae. Of these, 32 cells yielded stable membrane potential records with balanced electrodes about 1 min after impalement. Many cells lysed upon impalement.

14. A $t$ value was used to determine the significance of the effect of group (before dawn or after dawn) in a multiple linear regression model with SPSS/ $\mathrm{PC}+$ (SPSS Inc.), with the change in membrane potential as the dependent variable and current and group as independent variables.

15. Criteria for stability used for isolated cells were the same as for cells dispersed on cover slips. We attempted recordings from 155 cells from 172 retinae. Of these, 55 cells yielded stable membrane potential records.

16. We thank F. Strumwasser, B. Kellams, S Schacher, S. Dryer, and L. Latham for instruction or assistance with cell culture techniques and $W$. $O$. Friesen and T. Page for reviewing an early version of the manuscript. Supported by NIH grant NS15264 to G.D.B. S.M. was supported by the Deutsche Forschungsgemeinschaft and the NSF Center for Biological Timing; M.E.G. received support from NIH grant NS08806; and J.J.Z. was supported by the Undergraduate Research Program of the NSF Center for Biological Timing.

23 September 1992; accepted 13 November 1992

\title{
Tyrosine Phosphorylation of Actin in Dictyostelium Associated with Cell-Shape Changes
}

\author{
Peter K. Howard, Bartholomew M. Sefton, Richard A. Firtel*
}

When Dictyostelium cells that have initiated their developmental program upon starvation are returned to growth medium, there is a rapid and transient de novo tyrosine phosphorylation of a 43-kilodalton protein. This protein was found to be actin. Most of the phosphorylation occurred in a single, minor acidic isoform of actin. Developing cells that had been returned to growth medium lost their pseudopod extensions, became round, and had reduced adhesion to the substratum. These effects occurred with kinetics that matched the increase in tyrosine phosphorylation of actin. In mutant cell lines in which the gene for the phosphotyrosine phosphatase PTP1 had been disrupted, tyrosine phosphorylation of actin was rapid and more prolonged. These cells responded with proportionally accelerated kinetics of cell rounding. Cell lines overexpressing PTP1 had diminished amplitude and duration of actin tyrosine phosphorylation and exhibited diminished cell-shape change and an accelerated return to the extended cell-shape morphology seen in starved cells.

A variety of extracellular signals, such as growth factors and chemoattractants, and intrinsic events, such as progression through the cell cycle, lead to changes in cell shape and altered filamentous (F)-actin (1). Changes in actin and the cytoskeleton occur in Dictyostelium after stimulation of cells by the chemoattractant adenosine $3^{\prime}, 5^{\prime}$-monophosphate (cAMP) (2). Binding of cAMP to surface receptors leads to polymerization of actin within seconds and subsequent changes in the association of actin binding proteins with actin and the cytoskeleton. These responses

P. K. Howard and R. A. Firtel, Department of Biology Center for Molecular Genetics, University of California, San Diego, La Jolla, CA 92093

B. M. Sefton, Molecular Biology and Virology Laboratory, The Salk Institute for Biological Sciences, San Diego, CA 92186

*To whom correspondence should be addressed. lead to specific morphological changes within the cell and to extension of pseudopodia in the direction of the emitted cAMP signal. Little is known, however, about the signal transduction steps that connect the initial signaling events to the morphological changes. Changes in the $\mathrm{Ca}^{2+}$ concentration are thought to regulate some actin binding protein associations with the cytoskeleton (3), and in Dictyostelium the 1,2-diacylglycerol stimulates actin polymerization by apparent de novo formation of actin nucleation sites (4). We provide evidence that changes in the tyrosine phosphorylation of actin correlate with changes in the cell shape in Dictyostelium.

Changes in protein tyrosine phosphorylation have been examined through the Dictyostelium developmental cycle by protein immunoblotting with antibodies to 